\title{
Visión histórica de la respuesta a las amenazas naturales en Chile y oportunidades de gestión del riesgo de desastre $^{1}$
}

\author{
Pablo Camus², Federico Arenas 3 , Marcelo Lagos ${ }^{4}$ y Andrés Romero ${ }^{5}$
}

\begin{abstract}
RESUMEN
Las condiciones geográficas chilenas hacen del país un escenarío donde permanentemente se dejan sentir las denominadas amenazas naturales, poniendo a prueba de manera casi constante la capacidad de la sociedad chilena para levantarse luego de cada evento y de mejorar sus formas de ocupación del espacio. El predominio de un esquema de funcionamiento basado casi exclusivamente en la rentabilidad y muy poco en los riesgos de localización, permite que una buena parte de nuestros asentamientos e instalaciones presenten niveles de vulnerabilidad importantes frente a ciertas amenazas naturales. El objetivo de este trabajo es hacer una revisión histórica del tema, buscando extraer las lecciones necesarias para establecer formas más sustentables de construcción del espacio geográfico chileno.
\end{abstract}

Palabras clave: Reducción del Riesgo de Desastres, Gestión del Riesgo de Desastres, Desarrollo Local, Resiliencia.

\begin{abstract}
The geographical conditions in Chile make it a country that persistently experiences natural hazards, constantly testing the capacity of the Chilean society to pull itself up after every event and to improve the ways in which they occupy their territorial space. The prevalence of zoning that focus almost exclusively on profitability and places little attention on localized risks, has allowed a majority of settlement projects and facilities to be built in areas that face significant risk due to natural hazards. The aim of this work is to present a historical review of the subject, seeking to illustrate previous experiences in order to establish more sustainable forms of building within the Chilean geographical space.
\end{abstract}

Key words: Disaster Risk Reduction, Disaster Risk Management, Local Development, Resilience.

1 Esta publicación contó con el apoyo del Centro Nacional de Investigación para la Gestión Integrada de Desastres Naturales (CIGIDEN), CONICYT/ FONDAP/15110017. Artículo recibido el 18 de diciembre de 2015, aceptado el 4 de mayo de 2016 y corregido el 16 de junio de 2016.

2 Instituto de Historia, Pontificia Universidad Católica de Chile (Chile). E-mail: pcamusg@uc.cl
3 Instituto de Geografía, Pontificia Universidad Católica de Chile (Chile) / Centro Nacional de Investigación para la Gestión Integrada de Desastres Naturales, CONICYT/FONDAP/15110017 (Chile). E-mail: farenasv@uc.cl

4 Instituto de Geografía, Pontificia Universidad Católica de Chile (Chile). E-mail: mlagoslo@uc.cl

5 Oficina Nacional de Emergencia (Chile). E-mail: aromero.geo@gmail.com 
El territorío de Chile, dada su particular configuración física compuesta por ríos torrentosos, grandes diferencias de altitud en unas pocas decenas de kilómetros, variabilidad climática marcada tanto longitudinal como latitudinalmente, localización frente a una zona de subducción y sobre el denominado cordón de fuego del Pacífico, es naturalmente generador de espacios dinámicos y muchas veces extremadamente frágiles, en los cuales la tarea de la ocupación sustentable se hace cada vez más difícil como por ejemplo el poblamiento de la precordillera $y$, en particular, de los espacios litorales, los cuales se convierten en distintos momentos en una amenaza para la vida humana y sus creaciones.

El objetivo de este trabajo es mostrar la recurrencia de las amenazas naturales en Chile, revisar algunas de las respuestas institucionales y explorar las posibilidades de gestión del riesgo a partir de la legislación vigente. En este sentido, en el primer apartado se analiza la magnitud de las pérdidas y costos de las principales catástrofes acaecidas en Chile durante el siglo $X X$ y se realizan algunas reflexiones en torno a la idea de reducción del riesgo de desastre. En seguida se presenta una visión de las respuestas de la sociedad chilena a las distintas amenazas naturales, para concluir con algunas reflexiones en torno a las posibilidades de gestión que se han ido generando históricamente a través del entramado de una profusa legislación sectorial.

\section{Las amenazas naturales en Chile y algunos conceptos de reducción de riesgo de desastre}

La historia del desarrollo territorial chileno se ha visto marcada por grandes catástrofes, cuya génesis son eventos o fenómenos naturales extremos de origen geológico e hidrometeorológicos que han sido potenciados por las formas de ocupación y uso del territorío. El historiador Rolando Mellafe plantea, en este sentido, que la historia del "acontecer infausto en Chile", expresada a través de calamidades reiteradas a lo largo de los siglos, descubre las tensiones de una sociedad obligada a enfrentarse en un diálogo constante, consciente e inconsciente, con su entorno. Mellafe calculó un promedio de un terremoto cada ocho años desde 1582 a 1956, de los cuales un $62 \%$ fue con tsunami, es decir que cada chileno de los siglos XVI a XIX al menos vivió dos o tres terremotos en su vida y uno de mediados del siglo veinte, siete, si se considera el explosivo aumento de la esperanza de vida (Mellafe, 1994).

Sin ir más lejos, solo en el último siglo han ocurrido más de 60 eventos naturales con efectos desastrosos para la comunidad, dejando cerca de 45.000 personas fallecidas, y más de 12 millones de afectados (Cuadro $\left.N^{\circ} 1\right)$.

Cuadro $\mathrm{N}^{\circ} 1$

Principales desastres naturales desde 1906

\begin{tabular}{|l|r|r|r|r|}
\hline & \multicolumn{1}{|c|}{$\begin{array}{c}\text { Cantidad de } \\
\text { eventos }\end{array}$} & $\begin{array}{l}\text { Pérdidas mate- } \\
\text { riales en millo- } \\
\text { nes de USD }\end{array}$ & Fallecidos & $\begin{array}{r}\text { Población } \\
\text { Afectada }\end{array}$ \\
\hline Terremotos y tsunamis & 24 & 69.540 & 42.026 & 11.132 .251 \\
\hline Inundaciones & 19 & 1.207 & 1.066 & 1.342 .624 \\
\hline Remociones en Masa & 4 & 10 & 232 & 112.811 \\
\hline Erupciones Volcánicas & 7 & 103 & 110 & 89.850 \\
\hline
\end{tabular}

Fuente: Centro Sismológico Nacional de la Universidad de Chile (n.d.); EM-DAT (2014); INE (2005); Maddison (2010); U.S. Bureau of Labor Statistic (2014); World Bank (2012). 
En cuanto a cómo ha sido la distribución de estos fenómenos en el tiempo (Figura 1), se observa que los principales eventos son los sismos de Chillán (1939), Valdivia (1960), Illapel (1971), San Antonio (1985), Maule (2010) e Iquique (2014); los cuales, aunque de diferentes magnitudes, se distribuyen a lo largo de Chile de forma relativamente homogénea en el tiempo. Por otro lado, cabe señalar que a partir de los últimos 50 años comienzan a tomar importancia amenazas naturales más locales, asociadas a eventos hidrometeorológicos principalmente, entre ellos diversas inundaciones y remociones en masa, destacándose los aluviones de Antofagasta (1991), Quebrada de Macul (1993), y Atacama (2015).

Figura $\mathrm{N}^{\circ} 1$

Población afectada en desastres socionaturales desde 1906

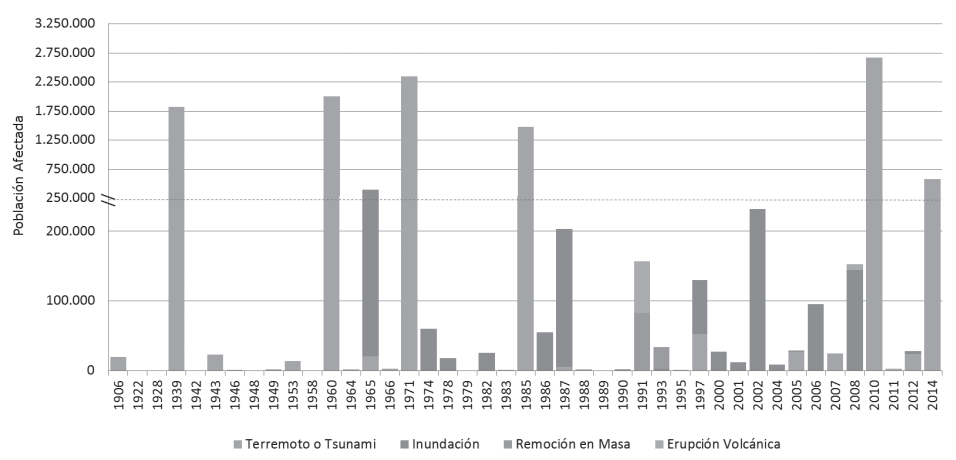

Fuente: Centro Sismológico Nacional de la Universidad de Chile (n.d.); EM-DAT (2014); INE (2005); Maddison (2010); U.S. Bureau of Labor Statistic (2014); World Bank (2012).

En relación con las pérdidas materiales, en el mismo período de tiempo se alcanzaron 70.800 millones de dólares (actualizados a valores del 2013) en daños -equivalentes al $70 \%$ del PIB de la Región Metropolitana del año 2012 (Banco Central de Chile, 2013)-, las cuales se concentran en daños relativos a terremotos y tsunamis. Cabe señalar que, a pesar que durante este período se promedia un sismo de magnitud 7 o superíor cada cinco años, a partir de 1971 el promedio de las pérdidas materiales baja considerablemente, tal como se puede ver en la Figura $N^{\circ} 2$.

Figura $N^{\circ} 2$

Pérdidas materiales en millones de dólares desde 1906

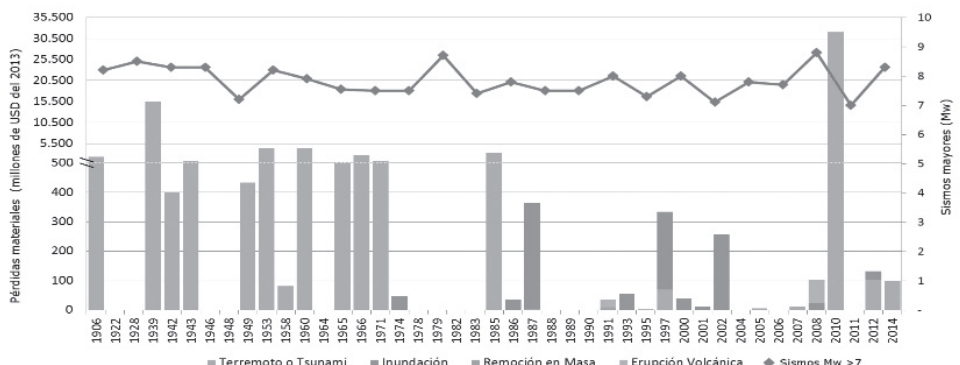

Fuente: Centro Sismológico Nacional de la Universidad de Chile (n.d.); EMDAT (2014); INE (2005); Maddison (2010); U.S. Bureau of Labor Statistic (2014); World Bank (2012) 
Tal como se puede observar en la Figura $\mathrm{N}^{\circ} 1$ y $\mathrm{N}^{\circ} 2$, y con más detalle en el Cuadro $N^{\circ} 1$, los principales desastres de origen natural de este último siglo son generados por terremotos y tsunamis, con 24 eventos que generaron el 99\% de las pérdidas humanas y materiales. Dentro de los eventos más significativos para la población y la economía del país se destacan el terremoto de Chillán de 1939, que originó pérdidas iguales al 55\% del PIB de Chile en ese año; el terremoto de Valparaíso de 1906, que alcanzó un 17\% del PIB en daños; y el terremoto del Maule 2010, con daños por un $14 \%$ del PIB.

Dichos eventos físicos, que aparentan ser naturales, en su esencia son creados por la intervención humana, ya que se gestan en la intersección de las actividades de la sociedad moderna, como la deforestación, los cambios en los patrones de uso del suelo y otros procesos sociales (Romero et al., 2010), con los procesos propios de la naturaleza, creando y/o ampliando las condiciones de riesgo. Es por esta razón que se les denomina riesgos socionaturales (Lavell, 2000).

En efecto, desde la década de los ochenta se han venido desarrollando distintos enfoques para entender los riesgos y desastres en América Latina desde una perspectiva más social. Cardona (2001) define el riesgo como el potencial de pérdidas que pueden ocurrirle al sujeto o sistema expuesto, resultado de la convolución de la amenaza y la vulnerabilidad. En donde la "convolución" -como concepto matemático- se refiere a la concomitancia y mutuo condicionamiento, de la amenaza y la vulnerabilidad. Es decir, no se puede ser vulnerable si no existe una condición de amenaza, y no se está expuesto si no se es vulnerable a la acción potencial que representa dicha amenaza. Así, pocos analistas se centran hoy en día en la idea de que la magnitud, intensidad o duración de los eventos físicos explican por sí solo el nivel de daño sufrido ante una amenaza. La tendencia ha sido la de encontrar una explicación en el conjunto de las condiciones económicas, sociales y ambientales existentes, lo cual confirma la necesidad de enfrentar estas condiciones de riesgo de forma íntegra, afrontando todos sus factores por igual (Calvo, 2014). Los riesgos, entonces, son construcciones sociales, derivadas de las modificaciones antrópicas al ambiente. En este sentido, la vulnerabilidad socioeconómica es clave para comprender las causas de los desastres, y entender que las consecuencias de estos, no son homogéneas, sino que se distribuyen según las condiciones de vulnerabilidad (Campos, 2015).

La Estrategia Internacional para la Reducción de Desastres de la ONU (2010) señala que para poder reducir de forma efectiva el riesgo de desastre, la gestión del riesgo como proceso político-social es fundamental, ya que mediante un conjunto de instrumentos, estrategias, políticas y mecanismos de intervención, se pueden enfrentar los distintos factores de riesgo. Dicha gestión tiene dos puntos de referencia temporal, los cuales tienen implicancias sociales, económicas y políticas totalmente distintas. La primera referencia es aquella que enfrenta a los factores de riesgo ya existentes, con ejemplos de sobra a lo largo de Chile. El segundo referente es el que habla del riesgo futuro, aquel que la sociedad construirá al promover nuevas inversiones en infraestructura, producción, asentamientos humanos, etc. (Lavell, 2001). Todo esto declara la existencia de dos tiempos para la gestión del riesgo: la gestión correctiva o compensatoria y la prospectiva.

La gestión correctiva o compensatoria tiene que ver con el enfrentamiento de las amenazas, mitigando las posible pérdidas sociales a través de preparativos, atención, sistemas de alerta temprana, entre otros; y atenuando las pérdidas económicas con obras de mitigación, seguros, protección ambiental, sistemas de alerta temprana agrícolas, y gestión ambiental. En esta etapa la atención de la emergencia, en la medida en que gestione exitosamente el riesgo, puede convertirse en una oportunidad para el desarrollo, en un puente con el desarrollo sostenible (Lavell, 2001; ONU-EIRD Las Americas, 2010).

La gestión prospectiva hace referencia a la gestión de riesgos futuros, los cuales buscan normar y/o controlar nuevos desarrollos mediante la planificación e inversión pública, específicamente con herramientas como el ordenamiento territorial, la planificación del desarrollo, la planificación sectorial y planificación financiera, las cuales se proyectan a futuro para la evitar la creación o recreación 
de nuevos riesgos (ONU-EIRD Las Américas, 2010).

Es en este punto donde se requiere de una voluntad política y de un alto grado de conciencia y compromiso con la reducción del riesgo por parte de todos los actores sociales (Lavell, 2001;). Participación que es enfatizada por la UNISDR en el Marco de Acción de Hyogo (ONU, 2005), actualizado por el Marco de Sendai de 2015 (ONU, 2015), donde se subraya la importancia que cada Estado tiene, en cuanto a "la responsabilidad de perseguir su propio desarrollo sostenible adoptando medidas eficaces para reducir los riesgos de desastre, en particular para la protección de la población que se halla en su territorío, la infraestructura y otros bienes nacionales contra el impacto de los desastres". En específico, el Estado como ente planificador del territorío, tiene atribuciones ya que los planes de ordenamiento territorial se constituyen como una herramienta útil para definir el tratamiento idóneo de las áreas expuestas a amenazas, dado que la ubicación de estas es el factor clave que determina el nivel de riesgo asociado a un peligro dado (ONU, 2001).

\section{Revisión histórica de las respuestas a las amenazas naturales en Chile}

Hasta el día de hoy la primera reacción ante los desastres ha sido refugiarse en la religión y la fe. En el pasado se consideraba que las catástrofes eran un castigo divino causado por la ira de Dios ante los pecados humanos por lo que la oración y la piedad podían atenuar sus efectos destructivos o al menos calmar los espíritus y miedos. Así, por ejemplo, el historiador Benjamín Vicuña Mackenna indica que el terremoto de 1647, denominado "el terremoto magno", aterró a la muchedumbre y morigeró no pocos de sus hábitos licenciosos, alteró visiblemente la arquitectura de la ciudad, imprimió el espíritu religioso característico de la sociedad de Santiago en la segunda mitad del siglo XVII y originó la procesión del Señor de Mayo, que hasta el día de hoy se realiza en las calles de Santiago (Vicuña Mackenna, 1926).

Otra respuesta a terremotos y tsunamis, más racional, ha sido la relocalización de ciudades, como, por ejemplo, Concepción tras el evento del 25 de mayo de 1751 y Chillán luego de la catástrofe de 1835. Más recientemente se ha respondido a través del estudio de los fenómenos sísmicos con la creación del Instituto Sismológico de Chile y del mejoramiento de los materiales de edificación (Poirier, 2015). Tras el terremoto de 1906, el ingeniero civil Hormidas Henríquez señaló la urgente necesidad de mejorar los materiales de construcción y utilizar el "concreto armado" al que consideraba "el material del futuro" (Henríquez, 1907). En 1929, como consecuencia del devastador terremoto de Talca de 1928, se aprobó la Ley Nº 4.563 sobre Construcciones Asísmicas, que estableció que "las municipalidades que cuenten dentro de su territorío con una ciudad de más de veinte mil habitantes, someterán al Ejecutivo, dentro del plazo de seis meses, un anteproyecto de transformación de esas ciudades. Sobre la base de este anteproyecto, el Presidente de la República ordenará confeccionar un proyecto definitivo, el cual, una vez aprobado, servirá para dar las líneas de edificación" (Arenas et al., 2010). Esta normativa sirvió de base para la promulgación de una Ley y Ordenanza General de Construcciones y Urbanización que finalmente fue aprobada en 1935. Este cuerpo legal institucionalizó las primeras normas de diseño y construcción antisísmicas en Chile y sentó las bases para el diseño de los primeros planes reguladores de uso del suelo de las ciudades chilenas.

Es probablemente la primera norma en el mundo que tiene conceptos de diseño basados en teorías dinámicas, espectros de aceleración y consideración de las características del suelo y fundación. Tenía disposiciones administrativas para regular el ejercicio profesional y asegurar el control independiente de los proyectos y las obras. Luego del terremoto de 1939, se iniciaron estudios para modificar la normativa, los cuales concluyeron en 1949 con la aprobación de una nueva ordenanza. En 1972, se aprobó la norma chilena $N^{\circ} 433$, de cálculo antisísmico de edificios, basada en la observación de los terremotos de las Melosas en 1958, Valdivia en 1960 y la Ligua en 1965.

A partir de 1976 y 1979, la eliminación de los controles de los colegios profesionales y de los municipios sobre el ejercicio de 
la ingeniería, además del sistema "Ilave en mano", permitió una acción más libre de las empresas constructoras. En 1986, Elías Arze indicaba que se habían originado prácticas nocivas tras la eliminación del control independiente de los proyectos por las municipalidades, que desde entonces se limitaban a recibir y archivar documentos. Por otra parte, el sistema "llave en mano" había creado un mercado de proyectistas contratados por los constructores, los cuales debían competir por mejorar las utilidades. El problema era que el uso indiscriminado de la competencia en base a honoraríos terminaba por asignar el proyecto al profesional que cobraba menos o que garantizaba menores costos, reduciendo el consumo de fierro redondo por metro cuadrado por ejemplo (Arze, 1986).

En cuanto a la fiscalización, a pesar de las atribuciones de la Dirección de Obras Municipales, con la instauración de revisores independientes y de revisores de proyectos de cálculo estructural, el Estado chileno había abdicado de su rol fiscalizador en materia de construcción bajo el supuesto de que el sector privado se tenía que hacer responsable de sus actos. La ley señalaba que el primer vendedor, inmobiliaria o constructora, debía garantizar por diez años la edificación vendida. Sin embargo, los arquitectos proyectistas, ingenieros calculistas, arquitectos revisores independientes, constructores, jefes de obras e inspectores técnicos no tenían por obligación certificar que la construcción se hiciese de manera correcta por lo que muchas veces establecían relaciones de clientelismo con las empresas. Sin fiscalización y sin responsabilidades claramente establecidas la norma bien podía quedar en letra muerta (Arze, 1986). Con todo, se puede señalar que las construcciones modernas han resistido bien los últimos terremotos primando más bien la responsabilidad, aun cuando no han faltado las fallas humanas y también los seres inescrupulosos que eluden la normativa.

En 1996 se actualizó la norma NCH433 de diseño sísmico a partir de la experiencia adquirida en los terremotos de 1985 en la zona central y 1995 en Antofagasta. El 2003 se oficializaron las normas técnicas $\mathrm{NCH} 2369$ de diseño sísmico de estructuras e instalaciones industriales y la $\mathrm{NCH} 2745$ de análisis y diseño sísmico de edificios con aislación sísmica. Después del terremoto del 2010 nuevamente se ha revisado y actualizado nuevamente la $\mathrm{NCH} 433$, para añadir mayores niveles de exigencias, sobre todo respecto de la clasificación de suelos (Sáenz, 2012).

En relación a la amenaza de tsunami, el Ministerío de Vivienda y Urbanismo publicó la NTM007, establecida mediante Res. Ex. $N^{\circ}$ 8952, de fecha 5/12/2011. Se trata de una norma técnica sobre diseño estructural para áreas de riesgo, pero es una norma indicativa que no es obligatoria de cumplir, es decir, una parte de la gestión del riesgo de tsunami continúa esperando en Chile un marco jurídico normativo.

Las erupciones volcánicas ya se encuentran descritas en los trabajos de los naturalistas decimonónicos. A partir de la década de 1960 se han editado innumerables publicaciones que han permitido identificar diversas zonas de riesgo volcánico, caracterizar los procesos eruptivos, las condiciones tectónicas y magmáticas que los controlan, su evolución geológica, permitiendo establecer la historia eruptiva y los ciclos de actividades de diferentes volcanes. El 2 de marzo de 1964, por ejemplo, el volcán Villarrica entró en actividad provocando una violenta erupción que costó la vida de veintidós personas y arrasó con el poblado de Coñaripe, el cual fue fundado nuevamente unos mil metros más al sudeste del pueblo antiguo. Santiago es también una ciudad altamente vulnerable pues se localiza a los pies de los centros eruptivos activos del Tupungato-Tupungatito, San José-Marmolejo y Maipo-Diamante. El 29 de noviembre de 1987 la activación del volcán Tupungatito provocó la avalancha del río Parraguirre-Colorado que generó importantes daños materiales y la muerte de cuarenta y tres personas (Urrutia, 1993). Desde entonces han ocurrido diversas erupciones entre las que destacan la de los volcanes Lonquimay, Hudson, Chaitén, y recientemente del Villarrica y el Calbuco (2015). En este contexto, se creó del Servicio Nacional de Geología y Minería que desde 1996, entre otras labores, trabaja elaborando cartas que incluyen la actividad volcánica histórica, probabilidad de ocurrencia y trayectoria crítica de diversos volcanes de Chile, y estableciendo un sistema de monitoreo volcánico con el objetivo de 
anticiparse a los eventos y decretar la evacuación de los habitantes en riesgo.

En relación a las amenazas provenientes de eventos climáticos extremos como sequías, inundaciones y aludes, y dada la estrecha relación de los asentamientos humanos con los ríos o más bien torrentes del territorío nacional, tanto los ciclos húmedos como los ciclos secos han tenido consecuencias catastróficas para la población. En la zona central del país, donde se ubica la mayoría de la población, la concentración de las lluvias en períodos cortos de tiempo y las fuertes pendientes cordilleranas favorecen los desbordes de ríos y canales, además de los deslizamientos de tierra. Desde 1912 a 1985, solamente los deslizamientos habrían significado aproximadamente 989 muertos, 888 heridos, 4.031 familias damnificadas, 1.500 animales muertos, 285 casas dañadas y un número indeterminado de terraplenes, caminos y vías de comunicación cortados (Espinoza et al., 1985).

Además de las rogativas por el cese de las Iluvias o bien por su muy deseada llegada, las respuestas institucionales a las amenazas asociadas a fenómenos hidrometeorológicos están relacionadas con la construcción de obras físicas de infraestructura, tales como trabajos de defensa fluvial o bien canales, represas y estanques de regadío. Un ejemplo es la ciudad de Santiago y el Mapocho. Transformado en el "azote de la ciudad", pronto los gobiernos coloniales debieron ocuparse de las inundaciones de Santiago. En 1609, el gobernador García Ramón ordenó al agrimensor Ginés de Lillo la construcción de tajamares con el objetivo de evitar los continuos desbordes del Mapocho. En 1662, se aprobó un Impuesto de Tajamares con el objetivo de mantener y reparar estas obras. Los tajamares de Lillo fueron destruidos por las inundaciones del 30 de abril de 1748. Fueron reconstruidos por el gobernador Ortiz de Rosas y destruidos nuevamente en 1783. Una década después se reconstruyeron por iniciativa de Ambrosio O'Higgins y Joaquín Toesca. Fueron destruidos nuevamente en 1877. A fines del siglo XIX se iniciaron nuevas obras de contención del río (Castillo, 2014). Hasta 1980, a pesar del crecimiento en extensión de Santiago, solo se habían construido cinco quilómetros de tajamares en el tramo Quinta
Normal - Providencia. Las inundaciones de 1982 dieron un nuevo impulso a las obras completándose diez kilómetros más. Estas obras en todo caso no garantizan que el Mapocho no se desborde pues están proyectadas para resistir crecidas de hasta 1.200 metros cúbicos por segundo, las cuales podrían ser sobrepasadas una vez cada cien años.

Respecto de la amenaza de sequías, en 1726, el gobernador Gabriel del Cano Aponte propuso la idea de construir un canal que uniese el río Maipo con el Mapocho. El proyecto fue replanteado por Ambrosio O'Higgins e inaugurado un siglo después de la propuesta de Cano Aponte, irrigando de este modo el agreste y árido valle que separaba ambos caudales. En el siglo XIX se iniciaron una serie de proyectos privados con el objetivo de ampliar las zonas irrigadas y responder a las crecientes demandas de productos agrícolas. Así se construyeron los canales Waddington en el Aconcagua y Buzeta en el Choapa, además de los canales Pirque, Espejo, Ochagavía, Santa Rita, Viluco, Paine, Quinta, Culiprán y Puangue en el río Maipo. Las Mercedes y Mallarauco en el Mapocho. Lucano, Nuevo Cachapoal, Comunidad, Codao - Las Cabras, Almahue y Pichidegua en el río Cachapoal. La Punta, Común, Chimbarongo y Huique en el río Tinguiririca. Población y Santa Cruz en el estero Chimbarongo. Purísima, Cumpeo y Pelarco en el río Lontué. Además de Duao-Zapata, el Morro y Colbún en el río Maule. A fines del siglo XIX y principios del siglo XX, se iniciaron algunas iniciativas estatales como la ejecución de obras en Laguna del Huasco y la construcción de los canales Mauco, Maule, Melado y Laja. En 1929 se aprobó la Ley General de Regadío que emprendió un Plan Extraordinarío de Obras de Riego que incorporó hasta 1969 un total de 303.827 hectáreas. En la década de los setenta del siglo XX la inversión de la Dirección de Riego del Ministerío de Obras Públicas estuvo determinada por la necesidad de concluir con las obras iniciadas durante los gobiernos anteríores y en la de los ochenta en la reparación de las obras de riego existentes. La ley $\mathrm{N}^{\circ} 18.450$ de octubre de 1985 fijó normas e incentivos para el fomento a la inversión privada en obras de riego y drenaje, estableciendo un subsidio de hasta un $75 \%$ a las obras particulares que postulasen a los fondos estatales. Esta legislación inhibió 
al Estado de la iniciativa de proyectar obras mayores de riego al traspasar la iniciativa y el financiamiento al sector privado (Peralta, 1989).

Por otra parte, el Código de Aguas publicado en el Diarío Oficial el 29 de octubre de 1981 y el DFL № 1.123, publicado en el Diarío Oficial del 21 de diciembre de 1981, estableció normas sobre la ejecución de obras de riego y transformó el derecho de aprovechamiento, de ser un derecho real administrativo y esencialmente caducable, en un derecho civil, prácticamente sin limitación en términos de su utilización, posible de ser enajenado e hipotecado, independiente del contexto en que se hallaba inserto o del uso al que estuviera originalmente destinado. Empresas y particulares han hecho utilización de estas posibilidades que otorga la ley concentrando en sus manos parte importante de los derechos sobre las aguas de los ríos de Chile. Esta forma de tenencia ha introducido serias restricciones al momento de definir y acordar príoridades y alternativas de utilización de los recursos hídricos. Por otra parte, es evidente que la falta de coordinación del sinnúmero de entidades públicas y privadas que tienen algún tipo de tuición o injerencia sobre las cuencas hidrográficas y los cauces de los ríos impide una gestión sustentable del recurso. Los cauces de los ríos al ser terreno de muchos se han transformado en terrenos de nadie, donde más que una gestión integrada se suman acciones parciales que sin considerar debidamente sus impactos multiplican y malgastan los esfuerzos, y, además, sobreexplotan el recurso.

A nivel de uso de suelo y amenazas es interesante señalar también que el ciclo de exportación de trigo del agro chileno, que se extendió aproximadamente desde mediados del siglo XIX hasta la Gran Depresión de 1929, originó intensos procesos erosivos y probablemente un aumento de las inundaciones y de los cambios en las desembocaduras de los ríos y en los espacios litorales originados por la ampliación de la frontera agrícola y el denudamiento de los suelos de bosque nativo. Como un modo de detener estos procesos se incentivó la forestación de los suelos del país, por ejemplo, a través de la ley de bosques de 1931 que eximió de impuestos territoriales a los suelos forestados.
Más importante fue el Decreto Ley 701 de 1974 que bonificó con un $75 \%$ de los costos a los privados que emprendiesen proyectos de forestación. Esta política logró cubrir en 17 años un millón setecientas mil hectáreas en su gran mayoría con la especie pino insigne o radiata. El notable avance de las plantaciones de pino inició un intenso debate sobre el manejo de las cuencas hidrográficas en el país, pues mientras el sector productivo veía control de la erosión y emisión de oxígeno, los grupos ecologistas evidenciaban el desecamiento de las vertientes, la pérdida de biodiversidad, la acidificación de suelos y la expulsión de la población rural (Camus, 2006).

\section{La cuestión institucional y los desafíos del ordenamiento territorial}

La regulación del uso del suelo cumple un rol fundamental en la reducción del riesgo de desastre. Diferentes normas lo abordan. Sin embargo, la principal es la Ley y Ordenanza General de Urbanismo y Construcciones, Decretos Supremos 458 y 47, cuya reglamentación y fiscalización es responsabilidad del Ministerío de Vivienda y Urbanismo. Esta Ley establece los Instrumentos de Planificación Territorial, que en su elaboración exigen estudios fundados de riesgo, los que dependiendo de la peligrosidad de determinados agentes naturales, deben proponer zonas no edificables o áreas de riesgo. Sin embargo, la realidad demuestra un conjunto de dificultades que no han permitido implementar de forma adecuada el factor riesgo en la planificación territorial de Chile. Principalmente destaca la falta de voluntad, recursos y personal capacitado, capaz de solicitar y revisar estudios adecuados para la escala urbana. La consecuencia es que hoy existen numerosos asentamientos humanos expuestos a un conjunto de peligros potenciales (Lagos, 2010).

Adicionalmente, hay normas generales de ordenamiento territorial, las que sin contemplar instrumentos de planificación, regulan directamente la gestión del territorío a través de ciertos organismos. En el caso específico del ordenamiento territorial de uno de los espacios más frágiles en el caso chileno, el costero, la Ley de Concesiones Marítimas, sin establecer una planificación para el otor- 
gamiento de tales concesiones, regula la forma y requisitos para dicho otorgamiento, mediante decreto supremo emitido por la Subsecretaría de Marina. Además existen a la vez una serie de otras normativas sectoriales que regulan los requisitos para la instalación y el ejercicio de actividades específicas sobre el suelo o la explotación de este por parte de personas determinadas, como es el caso del Código de Minería, el Código de Aguas, la Ley de Navegación, la Ley General de Pesca y Acuicultura, el Reglamento de Deportes Náuticos, entre muchas otras.

El mapa de las responsabilidades institucionales que existe para la creación y ejecución de instrumentos de planificación territorial, no contribuye a facilitar las cosas. La administración pública, en general, está constituida por servicios públicos que se clasifican como centralizados, los que dependen directamente de la administración del Estado (ministeríos y servicios públicos generales), y descentralizados, los que legalmente constituyen personas jurídicas distintas del Estado y cuentan con patrimonio propio, sin perjuicio de lo cual sus políticas generales son definidas también por el poder central. Los organismos centralizados, para llevar a cabo sus funciones en todo el territorío nacional se subdividen territorialmente a distintas escalas (regional, provincial y comunal), denominando a tales reparticiones, servicios desconcentrados. Lo anteríor representa una gran dificultad a la hora de establecer instrumentos de planificación territorial, dado que ninguna de las instituciones sectoriales es responsable globalmente del tema del ordenamiento del territorío.

En la situación actual, la planificación del territorío en Chile no es integral sino más bien de compartimentos estancos, de acuerdo con las disposiciones que dictan los organismos sectoriales, y sin que estas sean necesariamente coordinadas. Los permisos ambientales, por ejemplo, se limitan por ahora a garantizar de manera exhaustiva, que cada uno de los organismos sectoriales constate el cumplimiento de sus requisitos propios, no asegurando así el uso adecuado del suelo en todas sus partes, sino que por segmentos y por actividades también separadas que se interfieren y superponen. La planificación territorial, que incluye la reducción de riesgo de desastre, debiera tener un carácter transversal. Actualmente no lo tiene generando problemas de coordinación y de coherencia en el uso del territorío. En este contexto, la planificación territorial y la gestión de la vulnerabilidad a las amenazas naturales se ve complicada por la baja articulación entre los distintos sectores que interactúan y la poca claridad en cuanto a las responsabilidades institucionales por niveles de intervención.

Con todo, el conocimiento sistemático y sistematizado, y la posible aplicación de la vasta legislación con incidencia territorial existente, representan a la vez una oportunidad de gestión y de reducción de riesgo de desastres, puesto que las decisiones en torno al desarrollo territorial y a la localización del equipamiento, de la infraestructura y otras áreas urbanas pueden ser debidamente respaldadas y apoyadas por los marcos jurídicos y normativos existentes.

Una planificación que incorpore lo jurídico y normativo que rige lo sectorial, como los cementeríos, las industrias, los vertederos, las urbanizaciones, legítima legal e institucionalmente la evaluación de la localización de infraestructura ordenadora del espacio urbano en el corto, mediano y largo plazo. Así, por ejemplo, la ubicación de los cementeríos condiciona la localización de infraestructuras de servicios básicos, como tuberías de agua potable y define el entorno inmediato como una zona de desarrollo urbano restringido. Más que mirar la dispersión institucional de la gestión territorial y de la reducción de riesgo de desastre como un obstáculo debe dimensionarse también que las instituciones y los marcos jurídicos existentes, bien conocidos y aplicados en forma coordinada, pueden transformarse en una reserva instrumental con un enorme potencial en la planificación y gestión de territorío y sus amenazas.

La evidente complejidad de la gestión del territorío y de la reducción del riesgo de desastre, dada por el grado de multisectorialidad existente, no es más que la constatación de la manifestación del problema de la superposición de funciones señalado. No, obstante, en la medida que se conozca e incorpore el marco jurídico sectorial a la planificación territorial, este adquirirá una connotación de normativa técnica de necesaria consideración 
a la hora de tomar decisiones de inversión en infraestructura, equipamiento o urbanización, transformándose en importantes instrumentos técnico jurídico ambientales. Existe un conjunto de leyes y decretos poco conocidos o no utilizados cuya aplicación puede potenciar la gestión territorial integral y la reducción de riesgo de desastre. El conocimiento y la correcta interpretación de las normativas con incidencia ambiental aplicables al desarrollo de los asentamientos humanos permitirían generar planes con mayor calidad técnica y mayor legitimidad.

\section{Conclusiones}

Entre los desafíos más importantes para Chile en los tiempos que vienen, se encuentra el tema de la adaptación fina de las formas de ocupación del espacio a las condiciones geográficas permanentes del país, esto es, dando cuenta de las distintas amenazas naturales y reduciendo el riesgo de desastres.

Del análisis retrospectivo de las principales iniciativas impulsadas en Chile para mejorar la gestión del riesgo de desastres, derivado de las amenazas naturales que afectan al país, se puede observar que una parte muy importante corresponde a medidas de carácter reactivo frente a las catástrofes y, más recientemente, que el Estado se ha desligado de ciertas tareas, al disminuir, por ejemplo, su participación en la construcción de obras de riego mayores, al traspasar al sector privado una serie de atribuciones relacionadas con la gestión y el uso de bienes comunes vitales como el agua, o también al relajar o disminuir los mecanismos de fiscalización del cumplimiento de la normativa antisísmica o al no implementar todavía una legislación indicativa que mejore nuestras capacidades de gestión del riesgo de tsunami.

La persistencia de un sistema sectorial y compartimentado de la planificación del desarrollo de los centros poblados y de los territoríos del país impone la necesidad de encontrar y utilizar márgenes e intersticios normativos y legales que permitan avanzar en una planificación flexible e integrada, que incorpore y coordine intersectorialmente todo el potencial de los actuales compartimentos estancos con incidencia en la gestión del riesgo, generando así escenaríos e instrumentos de planificación y ordenamiento territorial más allá de lo sectorial.

Actualmente existen diversos instrumentos jurídicos normativos sectoriales que, incorporados a los procesos de planificación y ordenamiento del territorío, pueden constituirse en instrumentos de gestión territorial que eventualmente permitirían disminuir la vulnerabilidad de los asentamientos humanos ante las amenazas naturales. Es innegable que la sola integración y aplicación de la normativa con incidencia territorial existente actualmente, constituiría un avance en la planificación y el ordenamiento del territorío y, en consecuencia, en la reducción del riesgo de desastre.

Es probable que el exagerado rol entregado al mercado en las últimas décadas, como prácticamente único "asignador" de recursos en el territorío - por encima de cualquier otra consideración ligada a criteríos de planificación territorial-, sea lo que explique por qué muchos de los asentamientos humanos y de las instalaciones de infraestructura crítica, presentan niveles de vulnerabilidad importantes frente a ciertas amenazas naturales. Lo anteríor pone en evidencia la necesidad de discutir criteríos de localización para ciertas actividades o discutir sobre cuáles son los sitios más adecuados para instalar actividades o infraestructuras consideradas críticas. La tarea queda planteada.

\section{Referencias bibliográficas}

ARENAS, F.; LAGOS, M. e HIDALGO, $R$. Los riesgos naturales en la planificación territorial. En: CENTRO DE POLÍTICAS PÚBLICAS. Temas de Agenda Pública. Santiago de Chile: Pontificia Universidad Católica de Chile, 2010, N³9, p. 1-11.

ARZE, E. 58 años de ingeniería antisísmica en Chile. Revista Chilena de Ingeniería. 1986, No 395. Disponible en Internet: http://biblioteca.cchc.cl/

BANCO CENTRAL DE CHILE. Cuentas Nacionales de Chile, PIB Regional 2012. Santiago de Chile: Banco Central de Chile, 2013. 
CALVO, F. Algunas cuestiones sobre geografía de los riesgos. Scripta Nova. Revista Electrónica de Geografía y Ciencias Sociales, 2014, N 10. Disponible en Internet: http://www.ub.edu/geocrit/sn-10.htm

CAMPOS-VARGAS, M.; TOSCANA-APARICIO, A. y CAMPOS ALANÍS, J. Riesgo socionaturales: vulnerabilidad socioeconómica, justicia ambiental y justicia espacial. Cuadernos de Geografía. Revista Colombiana de Geografía, 2015, Vol. 24, № 2, p. 53-69.

CAMUS, P. Ambiente, bosques y gestión forestal en Chile, 1541-2005. Santiago de Chile: Centro de Investigaciones Barros Arana de la Dirección de Bibliotecas, Archivos y Museos-Lom Ediciones, 2006.

CARDONA, O. La necesidad de repensar de manera holística los conceptos de vulnerabilidad y riesgo. Una crítica y una revisión necesaria para la gestión. In: Work-Conference on Vulnerability in Disaster Theory and Practice. Wageningen 29-30 de junio de 2001, p. 1-18.

CASTILLO, S. El río Mapocho y sus riberas. Espacio público e Intervención urbana en Santiago de Chile (1885-1918). Santiago de Chile: Ediciones Universidad Alberto Hurtado, 2014.

CENTRO SISMOLÓGICO NACIONAL DE LA UNIVERSIDAD DE CHILE. Sismos Importantes y/o Destructivos (1570 a la fecha). Santiago de Chile. 2014. Disponible en Internet: http://www.sismologia.cl/links/terremotos/

CRED. EM-DAT. The OFDA/CRED. Database Centre for Research on the Epidemiology of Disasters (CRED). Bruselas: Gobierno de Bélgica, 2009. Disponible en Internet: http://www.emdat.be

ESPINOSA, G.; HAJEK, E. \& FUENTES, E. Aproximación histórica a los deslizamientos de tierra que han provocado desastres en Chile. Boletín de Medio Ambiente y Urbanización. 1985, Año 3, Nº 10, p. 7-9.

HENRÍQUEZ, H. El Terremoto de Valparaíso bajo su aspecto constructivo. Valparaíso: 1907.
INSTITUTO NACIONAL DE ESTADÍSTICAS (INE) y COMISIÓN ECONÓMICA PARA AMÉRICA LATINA Y EL CARIBE (CEPAL). Chile: proyecciones y estimaciones de población. Total País 1950-2050. Santiago de Chile: INECEPAL, 2005.

LAGOS, M. Análisis del Riesgo de Desastre en Chile. Santiago de Chile: DIPECHO/ UNESCO, 2010.

LAVELL, A. Desastres urbanos: una visión global. In: Centro Internacional Woodrow Wilson. U.S. Agencia para el Desarrollo Internacional. U.S. Universidad de Michigan. Programas de Becados para Estudios de Población. Asociación de Investigación y Estudios Sociales (ASIES). Michigan, 2000, p. 11-21.

LAVELL, A. Sobre la gestión del riesgo: apuntes hacia una definición. 2001. Disponible en Internet:

http://www.cridlac.org/cd/cd_inversion/ pdf/spa/doc15036/doc15036-contenido.pdf

MADDISON, A. Statistics on world population, GDP and per capita GDP, 1-2008 $A D$. Groningen: Maddison Project, University of Groningen, 2010. Disponible en Internet: http://www.ggdc.net/maddison

MELLAFE, R. Percepciones y representaciones colectivas en torno a las catástrofes en Chile: 1556-1956. En: MELLAFE, R. y LOYOLA, L. (editores). La Memoria de América Colonial. Santiago de Chile: Editorial Universitaria, 1994, p. 102-121.

ORGANIZACIÓN DE NACIONES UNIDAS (ONU). Menos vulnerabilidad, Menos Desastres. Ciudad de Panamá: Secretaría de la Estrategia Internacional para la Reducción de los Desastres de Naciones Unidas (EIRD/ ONU), 2001.

ORGANIZACIÓN DE NACIONES UNIDAS (ONU) - ESTRATEGIA INTERNACIONAL PARA LA REDUCCIÓN DE LOS DESASTRES (EIRD). Reducción del riesgo de desastres: Entendiendo el riesgo de desastres. Génova: ONU-EIRD, 2010. Disponible en Internet:

http://www.eird.org/wikiesp/index.php/ Entendiendo_el_riesgo_de_desastres 
ORGANIZACIÓN DE NACIONES UNIDAS (ONU) - OFICINA DE LAS NACIONES UNIDAS PARA LA REDUCCIÓN DEL RIESGO DE DESASTRES (UNISDR). Informe de la Conferencia Mundial sobre la Reducción de los Desastres. In: Conferencia Mundial sobre la Reducción de los Desastres. Kobe-Hyogo 18-22 de enero de 2005, p. 1-44.

ORGANIZACIÓN DE NACIONES UNIDAS (ONU). Marco de Sendai para la Reducción del Riesgo de Desastres 2015-2030. Génova: ONU-EIRD, 2015. Disponible en Internet: http://www.unisdr.org/files/43291_spanishsendaiframeworkfordisasterri.pdf

PERALTA, F. Ideas para la discusión de una política de riego, su aplicación al caso de Chile. Santiago de Chile: Ediciones Tacora Editorial Interamericana, 1989.

POIRIER, J.P. Fernand de Montessus de Ballore (1851-1923). París: Editions Hermann, 2015.

ROMERO, H.; FUENTES, C. y SMITH, P. Ecología política de los riesgos naturales y de la contaminación ambiental en Santiago de Chile: necesidad de justicia ambiental. Scripta Nova. Revista Electrónica de Geografía y Ciencias Sociales, 2010, Vol. XIV, N³31.
Disponible en Internet: http://www.ub.edu/ geocrit/sn/sn-331/sn-331-52.htm

SÁEZ DEL PINO, J. Normativa nacional antisísmica en materia de construcción. Bases y proyecciones. Santiago de Chile: Memoria para optar al Título Profesional de Abogado, Facultad de Derecho, Universidad de Chile, 2011.

U.S. BUREAU OF LABOR STATISTIC. Consumer Price Index Data from 1913 to 2014. Washington DC: United States Department of Labor. 2014. Disponible en Internet: http://www.bls.gov/data/inflation_calculator. htm

URRUTIA, R. y LANZA, C. Catástrofes en Chile: 1541-1992. Santiago de Chile: Editorial La Noria, 1993.

VICUÑA MACKENNA, B. Historia crítica y social de la ciudad de Santiago: 1541-1868. Santiago de Chile: Nascimiento, 1924-1926.

WORLD BANK. World Development Indicators 2012. Washington, DC: World Bank Group, 2012. Disponible en Internet:

https://openknowledge.worldbank.org/handle/10986/6014 